\title{
Sputum endothelin-1 level is associated with active pulmonary tuberculosis and effectiveness of anti-tuberculosis chemotherapy
}

\author{
XIANG WANG ${ }^{1-4}$, JINGQUN TANG $^{2}$, RANRAN WANG $^{2}$, CHEN CHEN $^{2}$, SHICHUAN TAN $^{2}$, \\ FENGLEI YU ${ }^{2}$, YONGGUANG TAO ${ }^{1,3,4}$ and YUNPING $\mathrm{LI}^{5}$
}

\author{
${ }^{1}$ Cancer Research Institute, Central South University, Changsha, Hunan 410078; ${ }^{2}$ Department of Thoracic Surgery, \\ The Second Xiangya Hospital, Central South University, Changsha, Hunan 410011; \\ ${ }^{3}$ Key Laboratory of Carcinogenesis and Cancer Invasion, Ministry of Education; ${ }^{4}$ Key Laboratory of Carcinogenesis, \\ Ministry of Health of Hunan Province, Changsha, Hunan 410078; ${ }^{5}$ State Key Laboratory of Medical Genetics, \\ The Second Xiangya Hospital, Central South University, Changsha, Hunan 410078, P.R. China
}

Received November 2, 2014; Accepted October 27, 2015

DOI: $10.3892 /$ etm.2016.2980

\begin{abstract}
Pulmonary tuberculosis (TB) is a major global health problem. Endothelin (ET)-1 is an important pro-inflammatory factor in the airways, which acts as a chemoattractant and an upregulator of other inflammatory mediators. In the present study, the association of the sputum ET-1 level with active pulmonary TB and the effectiveness of anti-TB chemotherapy was explored for the first time. A total of 56 newly diagnosed patients with active pulmonary TB, 56 age- and gender-matched TB-free controls, and 43 subjects with latent TB were recruited to the study. Patients in the active TB group received standard anti-TB chemotherapy. Sputum samples were collected from all study subjects at baseline (day 0) and on days $1,2,4,6,10$ and 14 of treatment for the active TB group and the ET-1 level was determined by enzyme-linked immunosorbent assay. The sputum ET-1 level in the active TB group was significantly higher than those in the latent TB and the non-TB groups at baseline. Following adjustment for confounders such as age, gender, severity of clinical presentation, plasma ET-1 level and comorbidities that might affect the sputum ET-1 level, multivariate logistic regression analysis revealed that sputum ET-1 level was an independent indicator for active pulmonary TB. In the active TB group during anti-TB chemotherapy, decrements in the sputum ET-1
\end{abstract}

Correspondence to: Dr Yunping Li, State Key Laboratory of Medical Genetics, The Second Xiangya Hospital, Central South University, 139 Middle Renmin Road, Changsha, Hunan 410078, P.R. China

E-mail: amyli@csu.edu.cn

Dr Yongguang Tao, Cancer Research Institute, Central South University, 110 Xiangya Road, Changsha, Hunan 410078, P.R. China E-mail: taoyong@csu.edu.cn

Key words: endothelin-1, tuberculosis, pulmonary tuberculosis, sputum, biomarker level were in significant correlation with decrements in the number of colony-forming units and increments in the time to positivity in a Mycobacteria Growth Indicator Tube assay. In conclusion, this study indicates that an elevated sputum ET-1 level is an independent indicator of active pulmonary TB and suggests that decrements in the sputum ET-1 level could reflect the effectiveness of anti-TB chemotherapy.

\section{Introduction}

Although various treatments for pulmonary tuberculosis (TB) are available, TB remains a major health problem worldwide, with an estimated 8.6 million new cases and 1.3 million mortalities in 2012 (1). The World Health Organization estimates that one-third of the global population has latent Mycobacterium tuberculosis (M. tuberculosis) infection, among which 5-10\% are likely to develop active TB during their lifetime (1). A rapid and accurate diagnosis test for active TB would enable the early treatment of this disease and reduce transmission, thereby facilitating TB control (2). However, the diagnostic tests currently available have significant deficiencies, such as a lack of sensitivity and specificity (3). In addition, a marker that precisely reflects the effectiveness of antimicrobial therapy would be useful in assessing the response to anti-TB treatments.

Endothelin (ET)-1 is a potent vasoconstrictor that exerts various effects in the respiratory tract (4), including the stimulation of mucus secretion, airway edema, smooth muscle mitogenesis and bronchial hyperresponsiveness (5). In addition, it is considered to have important pro-inflammatory effects in the airways, where it acts as a chemoattractant and also upregulates other inflammatory mediators such as interleukin (IL)-6, IL-8 and granulocyte-macrophage colony-stimulating factor (GM-CSF) (4). ET-1 is produced by human airway epithelial and endothelial cells and macrophages (4). Sputum levels of ET-1 have been reported to increase in patients with chronic obstructive pulmonary disease (COPD) during exacerbation (5). In addition, sputum ET-1 levels are also elevated in patients with cystic fibrosis and COPD compared with the levels in normal subjects (6). 
Our pilot study suggested that elevated sputum ET-1 levels might indicate active disease in patients with pulmonary M. tuberculosis infection. In the present study, the association of the sputum ET-1 level with active pulmonary TB and the effectiveness of anti-TB chemotherapy were explored.

\section{Materials and methods}

Subjects. From December 2012 to December 2013, 56 newly diagnosed patients with active pulmonary $\mathrm{TB}, 56$ age- and gender-matched non-TB controls, and 43 subjects with latent TB were recruited at the Second Xiangya Hospital of Central South University (Changsha, China). Diagnosis of active pulmonary TB was based on clinical symptoms, chest radiography, microscopy for acid fast bacilli and sputum M. tuberculosis culture. The inclusion criteria for patients with active TB were as follows: i) Had not received any anti-TB treatment prior to entering the study; ii) Culture or molecular confirmation of infection with drug-susceptible $M$. tuberculosis; and iii) human immunodeficiency virus (HIV) negative. The symptoms of pulmonary TB include fever, productive cough, night sweats, weight loss, chest pain and malaise. According to the severity of clinical presentation, patients with active TB were divided in three groups (mild, moderate and severe). Subjects with latent TB were those who had contact with a person with confirmed active TB and had a positive tuberculin skin test; none of them showed clinical symptoms or chest $\mathrm{X}$-ray signs suggesting active TB. The non-TB controls were those who had not contacted with any person with confirmed active TB and had a negative tuberculin skin test. All subjects in this study were HIV negative. Baseline characteristics of all subjects are shown in Table I. The study was approved by the Ethics Committee of the Second Xiangya Hospital. Written informed consent was obtained from all subjects.

Treatment. All patients with active pulmonary TB received standard anti-TB chemotherapy with a weight-adjusted fixed-dose of $55 \mathrm{mg} / \mathrm{kg}$ Rifafour e-275 (Sanofi-Aventis, Beijing, China) consisting of isoniazid, rifampin, pyrazinamide, and ethambutol. The treatment was administered on an inpatient basis.

Sputum sampling. For patients with active TB, first morning sputum samples were collected at baseline (day 0) and on days $1,2,4,6,10$, and 14 during Rifafour e-275 treatment. For subjects with latent TB and non-TB controls, induced sputum samples were collected within $72 \mathrm{~h}$ after enrollment, as previously described (7). The samples were centrifuged at $2,000 \mathrm{x}$ g for $10 \mathrm{~min}$ and the supernatant was collected. The ET-1 level in the supernatant was quantified with a sandwich enzyme-linked immunosorbent assay (ELISA) kit (DET100; R\&D Systems, Minneapolis, MN, USA) according to the manufacturer's instructions. For patients with active TB, an aliquot of the sputum sample was subject to log colony-forming unit (CFU) determination on 7H11 agar with Selectatab (polymyxin B, ticarcillin, amphotericin B and trimethoprim; Mast Group, Ltd., Bootle, Merseyside, UK) added. Log CFU determinations were performed on samples collected on days $0,1,2,4$, 6,10 and 14. A second aliquot was decontaminated with $1 \%$ $\mathrm{NaOH}-\mathrm{N}$-acetyl-L-cysteine, diluted with phosphate-buffered saline (PBS) and centrifuged at $4^{\circ} \mathrm{C}$ and 3,000 x g for $15 \mathrm{~min}$.

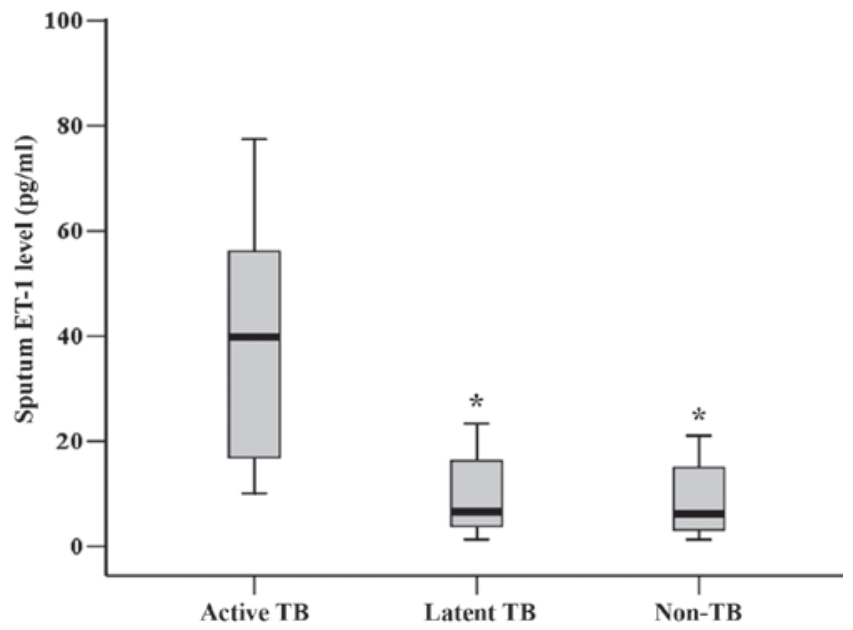

Figure 1. Sputum endothelin (ET)-1 levels in patients with active pulmonary tuberculosis (TB), subjects with latent TB, and non-TB controls Baseline sputum ET-1 levels in the three groups are represented as boxplots. ${ }^{*} \mathrm{P}<0.05$ vs. the active TB group.

The supernatant was discarded and the pellet resuspended in $1.5 \mathrm{ml}$ PBS. Then, $500 \mathrm{ml}$ of this suspension was used to inoculate a Mycobacteria Growth Indicator Tube (MGIT; BD Biosciences, Sparks Glencoe, MD, USA) supplemented with oleic acid, albumin, dextrose and catalase (OADC), and polymyxin $\mathrm{B}$, amphotericin $\mathrm{B}$, nalidixic acid, trimethoprim, azlocillin (PANTA). MGITs were incubated at $37^{\circ} \mathrm{C}$ in a BACTEC MGIT 960 instrument (BD Biosciences) until they were flagged positive, or for a maximum of 42 days if no growth was detected. Time to positivity (TTP) in MGIT culture was recorded. Contamination was excluded by placing one drop of positive liquid culture on a blood agar plate (NHLS, Cape Town, South Africa) and by incubating for $48 \mathrm{~h}$ at $37^{\circ} \mathrm{C}$ without visible growth.

Statistical analysis. Statistical analyses were performed using SPSS for Windows, version 13.0 (SPSS, Inc., Chicago, IL, USA). ET-1 levels were expressed as the median with interquartile range. Other continuous variables were expressed as mean \pm standard deviation. Comparisons of sputum ET-1 levels among subject groups were performed with nonparametric Kruskal-Wallis $\mathrm{H}$ tests followed by pairwise comparisons using Nemenyi tests. Categorical variables were compared with Chi-square tests. Correlation analyses between the changes in sputum ET-1 level and the changes in log CFU or TTP results were examined using Spearman's rank tests. Multivariate logistic regression was performed to assess the odds ratio (OR) and its $95 \%$ confidence interval (CI). A two-tailed $\mathrm{P}<0.05$ was considered statistically significant.

\section{Results}

Elevated sputum ET-1 level is associated with active pulmonary $T B$. As shown in Table I, there were no significant differences in age, gender and the prevalence of the co-morbidities hypertension, coronary artery disease, chronic bronchitis and COPD among the subject groups at baseline. The active TB group had a significantly higher sputum ET-1 level, but not plasma ET-1 level than the latent TB and the non-TB groups at baseline (Table I). 
Table I. Baseline characteristics of study subjects.

\begin{tabular}{|c|c|c|c|c|}
\hline Characteristic & Active TB $(\mathrm{n}=56)$ & Latent TB $(n=43)$ & Non-TB $(n=56)$ & P-value \\
\hline Age (years) $)^{\mathrm{a}}$ & $59.7 \pm 16.9$ & $55.2 \pm 18.5$ & $57.1 \pm 16.4$ & 0.83 \\
\hline \multicolumn{5}{|l|}{ Age group (years) } \\
\hline $15-29$ & $6(10.7)$ & $6(14.0)$ & $6(13.3)$ & \multirow[t]{4}{*}{0.95} \\
\hline $30-44$ & $7(12.5)$ & $6(14.0)$ & $7(13.3)$ & \\
\hline $45-59$ & $12(21.4)$ & $12(27.9)$ & $12(21.4)$ & \\
\hline$\geq 60$ & $31(55.4)$ & $19(44.1)$ & $31(55.4)$ & \\
\hline Age range (years) & $18-72$ & $17-69$ & $18-72$ & \\
\hline Male gender & $39(69.6)$ & $27(62.8)$ & $39(69.6)$ & 0.72 \\
\hline M. tuberculosis culture positivity & $41(73.2)$ & $0(0)$ & $0(0)$ & 1.00 \\
\hline \multicolumn{5}{|l|}{ Clinical presentation } \\
\hline Mild & $5(8.9)$ & - & - & \multirow[t]{3}{*}{-} \\
\hline Moderate & $40(71.4)$ & - & - & \\
\hline Severe & $11(19.6)$ & - & - & \\
\hline Sputum ET-1 level (pg/ml) & $39.7(17.9-56.4)$ & $6.3(3.1-16.7)^{\mathrm{a}}$ & $5.7(2.6-15.2)^{\mathrm{a}}$ & $<0.01$ \\
\hline Plasma ET-1 level (pg/ml) & $1.5(1.3-1.8)$ & $1.3(1.0-1.8)$ & $1.2(1.0-1.7)$ & 0.08 \\
\hline \multicolumn{5}{|l|}{ Co-morbidities } \\
\hline Hypertension & $27(48.2)$ & $19(44.2)$ & $24(42.9)$ & 0.84 \\
\hline CAD & $23(41.1)$ & $16(37.2)$ & $21(37.5)$ & 0.90 \\
\hline Chronic bronchitis & $17(30.4)$ & $11(25.6)$ & $10(17.9)$ & 0.30 \\
\hline COPD & $10(17.9)$ & $5(11.6)$ & $5(8.9)$ & 0.36 \\
\hline
\end{tabular}

Age is presented as the mean \pm standard deviation. Sputum and plasma ET-1 levels are presented as the median (interquartile range). Comparisons of sputum and plasma ET-1 levels among subject groups were performed with nonparametric Kruskal-Wallis H tests followed by pairwise comparisons using Nemenyi tests. All categorical variables are expressed as n (\%) and comparisons were performed with Chi-square tests. CAD, coronary artery disease; COPD, chronic obstructive pulmonary disease; TB, tuberculosis; M.tuberculosis, Mycobacterium tuberculosis . ${ }^{\text {ap }}<0.05$ vs. active TB.

Table II. Logistic regression analysis of factors significantly associated with sputum M. tuberculosis culture positivity.

\begin{tabular}{lccccc}
\hline Factor & $\begin{array}{c}\text { Point } \\
\text { estimate }\end{array}$ & $\begin{array}{c}\text { Standard } \\
\text { error }\end{array}$ & $\begin{array}{c}\text { Wald } \\
\text { Chi-square }\end{array}$ & P-value & $\begin{array}{c}\text { Odds } \\
\text { ratio }\end{array}$ \\
Severity of clinical presentation & 1.01 & 3.68 & 9.61 & $<0.01$ & 2.74 \\
fputum ET-1 level (pg/ml) & 1.87 & 0.49 & 4.17 & 0.04 & 6.50 \\
\hline
\end{tabular}

Multivariate logistic regression analysis was performed with sputum culture results $($ M. tuberculosis negative $=0$, . tuberculosis positive $=1)$ as the dependent variable. Age, gender (female=0, male=1), severity of clinical presentation (mild=1, moderate=2, severe=3), sputum ET-1 level, plasma ET-1 level, and co-morbidities (hypertension and/or coronary artery disease, no=0, yes=1; chronic bronchitis and/or COPD, no $=0$, yes=1) were used as independent variables. Severity of clinical presentation and sputum ET-1 level entered the logistic regression model. M. tuberculosis, Mycobacterium tuberculosis; ET, endothelin; CI, confidence interval.

As shown in Fig. 1, the sputum ET-1 level in the active TB group was significantly higher than those in the latent TB and the non-TB groups at baseline $(\mathrm{P}<0.01)$.

In order to identify the factors that significantly affected the sputum $M$ tuberculosis culture positivity, multivariate logistic regression analysis was performed using sputum culture results (M. tuberculosis negative $=0, M$. tuberculosis positive $=1$ ) as the dependent variable. Age, gender (female $=0$, male $=1)$, severity of clinical presentation (mild $=1$, moderate $=2$, severe $=3$ ), sputum
ET-1 level, plasma ET-1 level, and co-morbidities (hypertension and/or coronary artery disease, no $=0$, yes $=1$; chronic bronchitis and/or COPD, no=0, yes $=1$ ) were used as independent variables. As shown in Table II, the severity of clinical presentation and the sputum ET-1 level entered the logistic regression model. The results indicated that the severity of clinical presentation $(\mathrm{OR}=2.74,95 \% \mathrm{CI}=1.04-7.22, \mathrm{P}<0.01)$ and the sputum $\mathrm{ET}-1$ level $(\mathrm{OR}=6.50,95 \% \mathrm{CI}=1.32-32.02, \mathrm{P}=0.04)$ were significantly associated with sputum $M$.tuberculosis culture positivity, which 
Table III. Correlation between changes in sputum ET-1 level and changes in $\mathrm{CFU} / \mathrm{ml}$ in patients receiving anti-tuberculosis chemotherapy.

\begin{tabular}{ccr}
\hline Day & Correlation coefficient (r) & P-value \\
\hline 1 & 0.44 & $<0.01$ \\
2 & 0.51 & $<0.01$ \\
4 & 0.31 & 0.02 \\
6 & 0.36 & $<0.01$ \\
10 & 0.42 & $<0.01$ \\
14 & 0.54 & $<0.01$ \\
\hline
\end{tabular}

Patients with active pulmonary TB were treated with a weight-adjusted fixed-dose of Rifafour e-275. Changes in the sputum ET-1 level ( $\mathrm{pg} / \mathrm{ml}$ ) and $\mathrm{CFU} / \mathrm{ml}$ from baseline (day 0) on days 1, 2, 4, 6, 10 and 14 were expressed as $\Delta$ Sputum ET- 1 and $\Delta \log C F U / m l$, respectively. Spearman rank tests were performed to analyze the correlation between $\Delta$ Sputum ET-1 and $\Delta \log \mathrm{CFU} / \mathrm{ml}$ at each time point. ET, endothelin; $\mathrm{CFU}$, colony-forming unit.
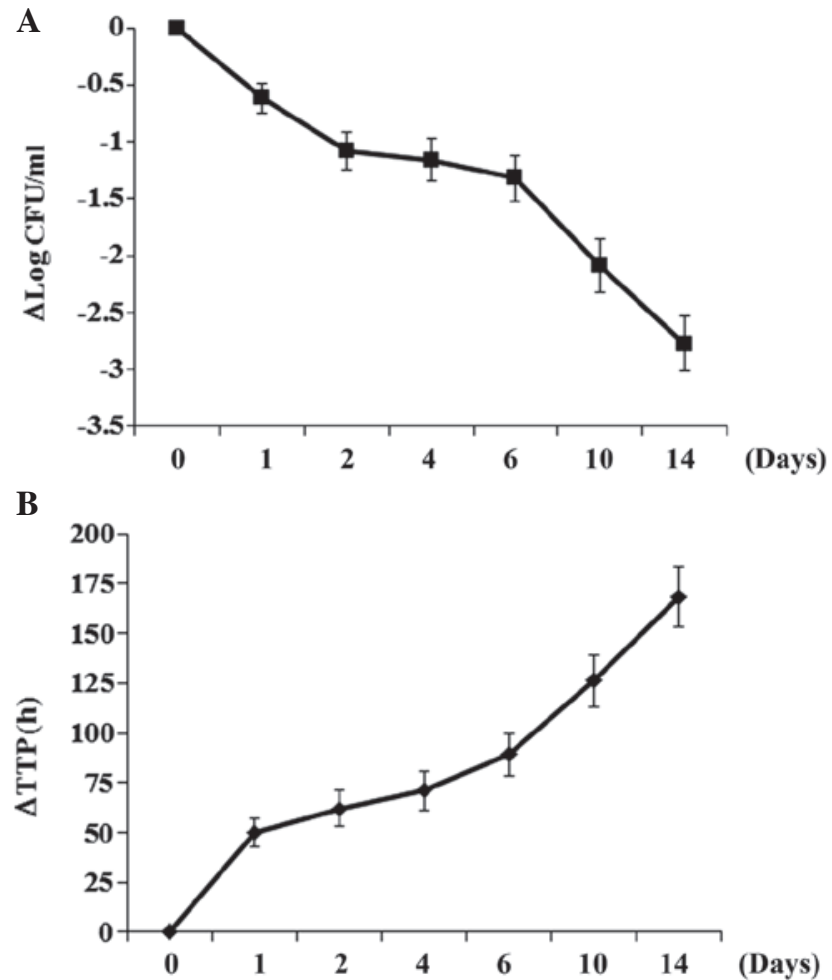

Figure 2. Changes in colony-forming unit (CFU) counts and time to positivity (TTP) in patients receiving anti-tuberculosis (TB) chemotherapy. Patients with active pulmonary TB were treated with a weight-adjusted fixed-dose of Rifafour e-275. Changes in CFU/ml and TTP (hours) from baseline (day 0 ) on days $1,2,4,6,10$ and 14 were expressed as (A) $\Delta \log \mathrm{CFU} / \mathrm{ml}$ and (B) $\Delta \mathrm{TTP}$ and plotted against time.

suggests that these two factors are independent indicators of active pulmonary TB.

Change in sputum ET-1 level correlates with patient response to anti-TB chemotherapy. In order to determine the association between the level of ET-1 in the sputum and the patient response
Table IV. Correlation between changes in sputum ET-1 level and changes in TTP in patients receiving anti-tuberculosis chemotherapy.

\begin{tabular}{ccc}
\hline Day & Correlation coefficient (r) & P-value \\
\hline 1 & -0.52 & $<0.01$ \\
2 & -0.42 & $<0.01$ \\
4 & -0.43 & $<0.01$ \\
6 & -0.46 & $<0.01$ \\
10 & -0.49 & $<0.01$ \\
14 & -0.56 & $<0.01$ \\
\hline
\end{tabular}

Patients with active pulmonary TB were treated with a weight-adjusted fixed-dose of Rifafour e-275. Changes in the sputum ET-1 level $(\mathrm{pg} / \mathrm{ml})$ and in TTP from baseline (day 0$)$ on days 1, 2, 4, 6, 10 and 14 were expressed as $\Delta$ Sputum ET-1 and $\Delta \mathrm{TTP}$, respectively. Spearman rank tests were performed to analyze the correlation between $\Delta$ Sputum ET-1 and $\Delta$ TTP at each time point. ET, endothelin; TTP, time to positivity.

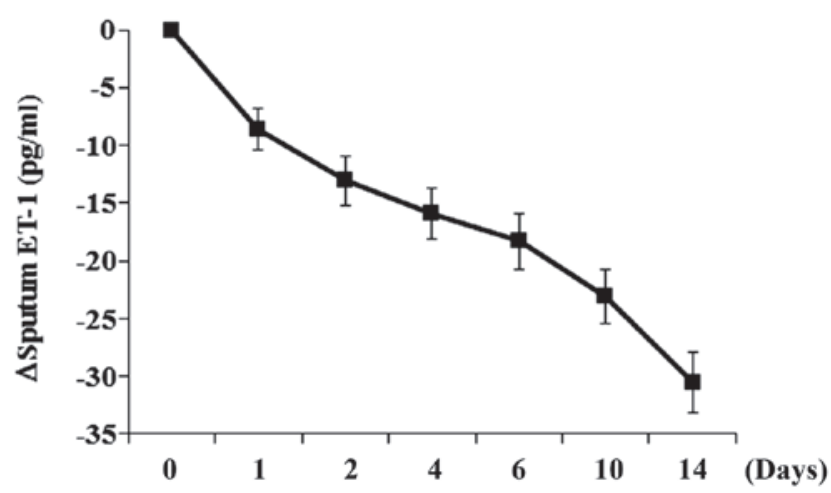

Figure 3. Changes in sputum endothelin (ET)-1 level in patients under anti-tuberculosis (TB) chemotherapy. Patients with active pulmonary TB were treated with a weight-adjusted fixed-dose of Rifafour e-275. Changes in the sputum ET-1 level (pg/ml) from baseline (day 0) on days 1, 2, 4, 6, 10 and 14 were expressed as $\Delta$ Sputum ET-1 and plotted against the time.

to anti-TB chemotherapy, patients in the active TB group were treated with a weight-adjusted fixed-dose of $55 \mathrm{mg} / \mathrm{kg}$ Rifafour e-275 and the sputum ET-1 level, the number of CFU/ml and TTP were measured at baseline (day 0 ) and on days 1, 2, 4, 6, 10 and 14. As shown in Fig. 2, the number of CFU and TTP decreased and increased, respectively, over the time of treatment. The sputum ET-1 level decreased over the time of treatment (Fig. 3), with a trend similar to that of the number of CFU. As shown in Table III, correlation analyses with Spearman rank tests revealed that decrements (from baseline) in sputum ET-1 level were in significant positive correlation with decrements (from baseline) in the number of CFU at each time point during the treatment, with the correlation coefficient ranging from 0.31 on day 4 to 0.54 on day 14 (all $\mathrm{P}<0.05$ ). By contrast, decrements (from baseline) in the sputum ET-1 level were in significant negative correlation with increments (from baseline) in TTP at each time point during the treatment, with the correlation coefficient ranging from -0.42 on day 2 to -0.56 on day 14 (all $\mathrm{P}<0.01$; Table IV). 


\section{Discussion}

The present study, to the best of our knowledge, provides the first evidence that the sputum ET-1 level is significantly associated with active pulmonary TB and the effectiveness of anti-TB chemotherapy.

ET-1, produced by airway epithelial and endothelial cells and macrophages (8-10), functions as a pro-inflammatory factor in the airways, where it acts as a chemoattractant and upregulates other important inflammatory mediators such as IL-6 and GM-CSF $(5,11)$. A systemic rise of ET-1 levels occurs in response to a variety of factors, including sepsis and ischemia $(5,12)$. In the present study, it was observed that the sputum ET-1 level was significantly elevated in patients with active pulmonary TB compared with patients with latent TB and TB-free controls. Following adjustment for confounders such as age, gender, severity of clinical presentation, plasma ET-1 level and comorbidities that might affect the sputum ET-1 level, multivariate logistic regression analysis revealed that the sputum ET-1 level was an independent indicator for active pulmonary TB. Since the plasma ET-1 level was not significantly increased, it is likely that the elevation of sputum ET-1 levels in patients with active pulmonary TB was due to the pulmonary, not systemic, inflammatory responses to active $M$. tuberculosis infection.

The viability of bacilli and the susceptibility to anti-TB therapy is usually monitored by culture (13), which remains the gold standard in the diagnosis and follow-up of mycobacterial infections. However, it is a time-consuming process, since $M$. tuberculosis grows slowly and several weeks or months are required for its detection in clinical samples (14). Inflammation-related factors have been suggested as potential biomarkers for active TB $(15,16)$. Travar et al $(15)$ reported that the sputum level of interferon $\lambda-2$ was significantly higher in patients with active pulmonary TB than in patients with latent TB and healthy controls. Cai et al (16) reported that the expression level of complement $\mathrm{Clq}$ in the peripheral blood was able to discriminate patients with active TB from those with latent TB infection and healthy controls. The results of the present study show that the sputum ET-1 level was significantly higher in the patients with active pulmonary TB than in those with latent TB and the TB-free controls. Whether these factors are connected in active pulmonary TB and how remain to be explored in our future studies.

In this study, decrements in the sputum ET-1 level significantly correlated with decrements in CFU and increments in TTP during anti-TB chemotherapy. This corroborates the finding that the sputum ET-1 level is significantly associated with active pulmonary TB, and also suggests that decrements in the sputum ET-1 level could be a potential indicator of the effectiveness of anti-TB chemotherapy. Determination of the sputum ET-1 level by ELISA is fast ( $<5 \mathrm{~h})$ and easy, which supports the feasibility of using the sputum ET-1 level as a biomarker for active pulmonary TB and the effectiveness of anti-TB chemotherapy. We plan to explore the clinical application value of the sputum ET-1 level for patients with active pulmonary TB in a future study with a large patient sample.

The present study has several limitations: i) Only HIV-negative subjects were enrolled to minimize the potential effects of immunodeficiency on the sputum ET-1 level, since ET-1 is profoundly involved in inflammatory responses in the airways. ii) Only newly diagnosed patients infected with drug-susceptible $M$.tuberculosis and without previous anti-TB treatment were enrolled in the active TB group to exclude possible confounding effects of drug-resistant $M$. tuberculosis on the effectiveness of anti-TB chemotherapy in this study (17). Nevertheless, the findings of this study provide a solid basis for future studies with a more extensive patient sample.

In conclusion, this study indicates that an elevated sputum ET-1 level is an independent indicator of active pulmonary TB and suggests that decrements in the sputum ET-1 level may reflect the effectiveness of anti-TB chemotherapy.

\section{References}

1. Global tuberculosis control: Surveillance, planning and financing. World Health Organization. Geneva, Switzerland, 2009.

2. Parashar D, Chauhan DS, Sharma VD and Katoch VM: Applications of real-time PCR technology to mycobacterial research. Indian J Med Res 124: 385-398, 2006.

3. Dorman SE: New diagnostic tests for tuberculosis: Bench, bedside, and beyond. Clin Infect Dis 50 (Suppl 3): S173-S177, 2010.

4. Zheng L, Tipoe G, Lam WK, Ho JC, Shum I, Ooi GC, Leung R and Tsang KW: Endothelin-1 in stable bronchiectasis. Eur Respir J 16: 146-149, 2000.

5. Roland M, Bhowmik A, Sapsford RJ, Seemungal TA, Jeffries DJ, Warner TD and Wedzicha JA: Sputum and plasma endothelin-1 levels in exacerbations of chronic obstructive pulmonary disease. Thorax 56: 30-35, 2001.

6. Chalmers GW, Macleod KJ, Sriram S, Thomson LJ, McSharry C, Stack BH and Thomson NC: Sputum endothelin-1 is increased in cystic fibrosis and chronic obstructive pulmonary disease. Eur Respir J 13: 1288-1292, 1999.

7. Bhowmik A, Seemungal TA, Sapsford RJ, Devalia JL and Wedzicha JA: Comparison of spontaneous and induced sputum for investigation of airway inflammation in chronic obstructive pulmonary disease. Thorax 53: 953-956, 1998.

8. Ehrenreich H, Anderson RW, Fox CH, Rieckmann P, Hoffman GS, Travis WD, Coligan JE, Kehrl JH and Fauci AS: Endothelins, peptide with potent vasoactive properties, are produced by human macrophages. J Exp Med 172: 1741-1748, 1990.

9. Nakano J, Takizawa H, Ohtoshi T, Shoji S, Yamaguchi M, Ishii A, Yanagisawa $\mathrm{M}$ and Ito $\mathrm{K}$ : Endotoxin and proinflammatory cytokines stimulate endothelin-1 expression and release by airway epithelial cells. Clin Exp Allergy 24: 330-336, 1994.

10. Giaid A, Polak JM, Gaitonde V, Hamid QA, Moscoso G, Legon S, Uwanogho D, Roncalli M, Shinmi O, Sawamura T, et al: Distribution of endothelin-like immunoreactivity and mRNA in the developing and adult human lung. Am J Respir Cell Mol Biol 4: 50-58, 1991.

11. Mullol J, Baraniuk JN, Logun C, Benfield T, Picado C and Shelhamer JH: Endothelin-1 induces GM-CSF, IL-6 and IL-8 but not G-CSF release from a human bronchial epithelial cell line (BEAS-2B). Neuropeptides 30: 551-556, 1996.

12. Warner TD and Klemm P: What turns on the endothelins? Inflamm Res 45: 51-53, 1996.

13. Takahashi $\mathrm{T}$ and Nakayama $\mathrm{T}$ : Novel technique of quantitative nested real-time PCR Assay for Mycobacterium tuberculosis DNA. J Clin Microbiol 44: 1029-1039, 2006.

14. Montenegro RA, Guarines KM, Montenegro LM, Lira LA, Falcão J, Melo FL, Santos FC, Nascimento AL, Zuzarte MS, Leite RC and Schindler HC: Assessment of messenger RNA (mRNA) of Mycobacterium tuberculosis as a marker of cure in patients with pulmonary tuberculosis. J Appl Microbiol 117: 266-272, 2014.

15. Travar M, Vucic M and Petkovic M: Interferon lambda-2 levels in sputum of patients with pulmonary Mycobacterium tuberculosis infection. Scand J Immunol 80: 43-49, 2014.

16. Cai Y, Yang Q, Tang Y, Zhang M, Liu H, Zhang G, Deng Q, Huang J, Gao Z, Zhou B, et al: Increased complement Clq level marks active disease in human tuberculosis. PLoS One 9: e92340, 2014

17. Mdivani N, Li H, Akhalaia M, Gegia M, Goginashvili L, Kernodle DS, Khechinashvili G and Tang YW: Monitoring therapeutic efficacy by real-time detection of Mycobacterium tuberculosis mRNA in sputum. Clin Chem 55: 1694-1700, 2009. 